\title{
Vertebral Pain in Acute COVID-19-Cases Report
}

\author{
Juraj Chochol ${ }^{1, *(\mathbb{D}}$, Andrej Džubera ${ }^{1}$, Róbert Illéš ${ }^{1}$, Alica Chocholová ${ }^{2}$ and Erika Zemková ${ }^{3,4}$ (i) \\ 1 Department of Neurosurgery, Faculty of Medicine of Slovak Medical University, \\ University Hospital—St. Michal's Hospital, 81108 Bratislava, Slovakia; andrej.dzubera@nsmas.sk (A.D.); \\ robert.illes@nsmas.sk (R.I.) \\ 2 Department of Pediatric Hematology and Oncology, National Institute of Children's Diseases, \\ Comenius University, 83340 Bratislava, Slovakia; alica.chocholova@nudch.eu \\ 3 Department of Biological and Medical Sciences, Faculty of Physical Education and Sports, \\ Comenius University in Bratislava, 81469 Bratislava, Slovakia; erika.zemkova@uniba.sk \\ 4 Faculty of Electrical Engineering and Information Technology, Sports Technology Institute, \\ Slovak University of Technology, 81219 Bratislava, Slovakia \\ * Correspondence: juraj.chochol@nsmas.sk; Tel.: +421-2-3261-2305
}

Citation: Chochol, J.; Džubera, A.; Illéš, R.; Chocholová, A.; Zemková, E. Vertebral Pain in Acute COVID-19-Cases Report. Appl. Sci. 2021, 11, 6926. https://doi.org/ $10.3390 /$ app 11156926

Academic Editor: Alessandro de Sire

Received: 3 July 2021

Accepted: 26 July 2021

Published: 28 July 2021

Publisher's Note: MDPI stays neutral with regard to jurisdictional claims in published maps and institutional affiliations.

Copyright: (c) 2021 by the authors. Licensee MDPI, Basel, Switzerland. This article is an open access article distributed under the terms and conditions of the Creative Commons Attribution (CC BY) license (https:/ / creativecommons.org/licenses/by/ $4.0 /)$.

\begin{abstract}
Pain is one of the most common complaints among patients infected by SARS-CoV-2. While headache and chest pain are reported widely among COVID-19 presentations, there are only scarce reports about vertebral pain. This study aims to describe cases of four healthy and physically active adults, in whom the new onset of back pain was the early or only sign of COVID-19 and the reason for seeking medical outpatient care. The vertebral pain showed great variability and changed dramatically during the course of disease. Pseudoradicular symptoms were present inconstantly and variably; in some patients they varied through the day from left to right side and irradiated from various sensory root segments. Clinical symptoms did not correspond with minor morphological changes presented on MRI scans. Our experience indicates that new onset of vertebral algic syndrome should be considered as an initial indicator of novel coronavirus infection in healthy and physically active adults. There is a pressing need for attention to be paid in the examination of patients with new vertebral pain onset in the coronavirus pandemic.
\end{abstract}

Keywords: SARS-CoV-2 infection; back pain; vertebral algic syndrome; first symptoms; case report

\section{Introduction}

Novel coronavirus infections were first documented in December 2019 in Wuhan (China) and spread rapidly worldwide, leading to the declaration of a global pandemic by the World Health Organization on 11 March 2020 [1]. The infection has been diagnosed in $179,261,894$ individuals, caused 3,884,666 deaths worldwide ([2], accessed on 23 June 2021), considerably affected all areas of everyday life, and caused large economic burdens.

Various manifestations of the severe acute respiratory syndrome coronavirus 2 (SARS$\mathrm{CoV}-2$ ) infection have been increasingly presented in the literature [3-5]. First, respiratory tract infection with lung involvement and severe respiratory distress syndrome was described [3-6]. As the pandemic spread, the diagnostic tools became widely available and the number of infected patients rose, with a wide variety of symptoms described.

From the data now available, it is known that the virus can invade different tissues and infection can exhibit numerous symptoms [7]. Primarily the respiratory tract is infected (nasal congestion, sore throat, cough, shortness of breath) [7,8]. The virus also has the ability to invade the neural system (headache, dizziness, cognitive disorders, various forms of psychic deterioration) [7-11], digestive tract (vomiting, diarrhea, abdominal pain) [5,7-10], cardiovascular system (chest pain, cardiac injury, thromboembolism) $[8,10,12]$, musculoskeletal system (joint pain, myalgia) [7-10,12], and also other body organs [9,10,12] and cause different pain manifestations $[7,8,10,12,13]$. The fever accompanying the infection might not be presented in mild, oligosymptomatic, or asymptomatic cases [7-9,12]. 
Nevertheless, a majority of patients infected with SARS-CoV-2 are asymptomatic or have only mild symptoms $[7,8,12]$. Some of the symptoms are unusual or even bizarre-e.g., loss of taste and smell [7,8,14], burning pain of skin [15], chest pain not corresponding to the site of lung involvement [16], various skin lesions [17]. There are still many unknowns regarding the relationships or processes of symptom development and their predictive value for the severity and outcome of the course of this disease $[6,9,18]$.

Pain is a commonly present non-specific symptom of viral infections, SARS-CoV-2 infection included. Muscle aches and pain are experienced by $15-59 \%$ of patients with coronavirus disease 2019 (COVID-19, the disease caused by SARS-CoV-2) [7,8,12,13,19]. However, the precise, direct, and indirect mechanisms of tissue damage and pain genesis in COVID-19 are not known and are the subject of worldwide research $[19,20]$. The mechanism of COVID19-induced pain remains unclear. Neuroinvasivity in human coronaviruses is known; the axonal transport is possibly used for propagation in neural involvement $[11,14,21,22]$. Pain occurs approximately 2 days before seeking medical attention $[12,16]$ and is a more common sign (69.3\%) [12] than loss of smell (43.5\%) [12] and taste (33.9\%) [14].

Among symptoms associated with COVID-19, less attention has been paid to vertebral pain. Low back pain or neck pain could be presented as nonspecific constitutional symptoms with other symptoms such as headache, fatigue, sore throat, cough, etc. $[7,12]$ or, based on our experience, also as the only or early sign in oligosymptomatic COVID-19.

Here we describe the cases of four patients presenting with vertebral algic syndrome with/without radicular or pseudoradicular irradiation in later PCR-confirmed SARS-CoV-2 infection. We highlight the pressing need for vigilance in the examination of patients with new onset of back pain during the pandemic. On the basis of case reports, we sought to lessen the gap in currently available literature by adding another mosaic stone to the knowledge of COVID-19 manifestation and provide a helping hand to other colleagues in the first line.

\section{Materials and Methods}

Four outpatient patients ( 3 men with low back pain and 1 woman with neck pain, age ranging from 32-45 years, mean age 39.5 years) later confirmed through positive PCR tests for SARS-CoV-2, were treated at the Department of Neurosurgery, Faculty of Medicine of Slovak Medical University, University Hospital—St. Michal's Hospital in Bratislava, Slovakia. Informed consent was obtained from all subjects.

Their subjective feelings of pain were evaluated retrospectively before infection, at time of onset during neurosurgical examination, and after recovery from COVID-19 with the visual analogue scale (VAS). Using a ruler, the VAS score was determined by measuring the distance on the 10-cm line between the "no pain at all" and "my pain is as bad as it could be". Each patient marked their score from 0-10. A higher score indicated greater pain intensity [23]. The retrospective analysis of routinely obtained data was performed. SARS-CoV-2 was PCR-confirmed during acute infection and the worsening of patient health status at accredited Slovak laboratories.

Cohen's $d$ was used to evaluate pre-post infection changes in VAS score. An effect size of less than 0.2 was considered small, approximately 0.5 was moderate, and greater than 0.8 was large [24]. Effect sizes were calculated with the software program $G^{*}$ power 3.1 for Mac OS X [25].

\section{Cases Report}

All subjects were physically active [26] with almost no previous history of vertebral algic syndrome or back/neck pain (VAS of $0.25 \pm 0.5$ ). They had normal weight and no other comorbidity.

At the time of neurosurgical examination, vertebral pain was the major complaint in all patients (VAS of $6.25 \pm 0.957$ ); no other clinical manifestation of COVID-19 infection was present. The infection of SARS-CoV-2 was later confirmed by PCR. The PCR tests were conducted for later-confirmed positive contact with infected person or as a periodically 
performed screening test of healthcare professionals (one from the group). None of the tests were indicated for suspected SARS-CoV2 infection.

The vertebral pain showed great variability; the intensity changed dramatically during the day and during the course of disease. The migrating pseudoradicular symptoms were present inconstantly and variably in the cervical or lumbar spine. In 2 patients, pseudoradicular irradiation radiated from various sensory root segments and changed the lateralization throughout the day (from left to right side, and vice versa). Relief positions were difficult to find. The neurological examination findings did not correspond to clinical symptoms or minor morphological changes presented on realized MRI scans (Figure 1a,b).

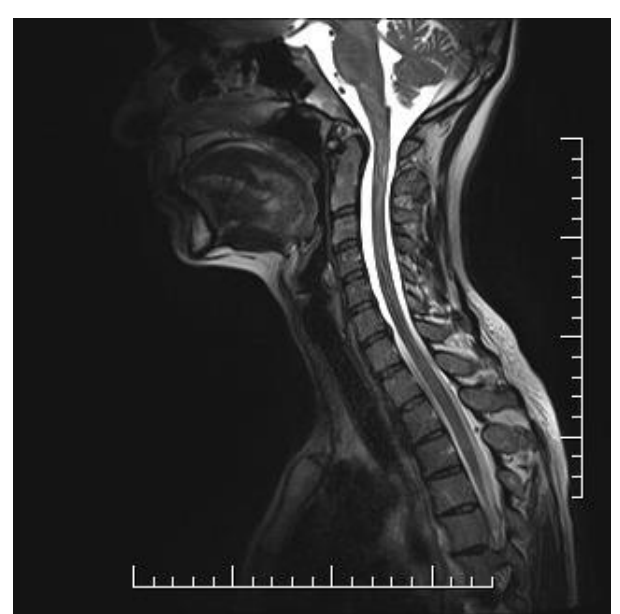

(a)

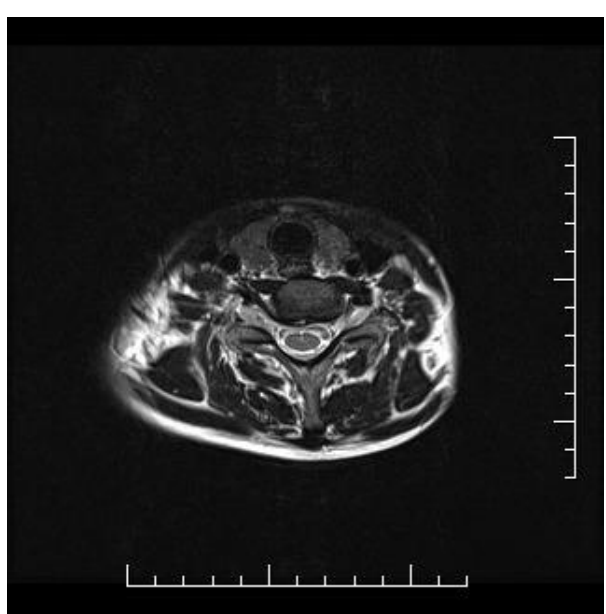

(b)

Figure 1. Minor morphological changes in MRI scan of cervical spine in 38-year-old woman with migrating pseudoradicular symptoms in COVID-19 without previous history of cervical vertebral algic syndrome. (a) Sagittal T2-weighted sequence; (b) Axial T2-weighted sequence.

All patients were afebrile or subfebrile during the entire course of disease with very mild or no upper respiratory tract symptoms (nasal congestion) and minor fatigue. Hospitalization was not necessary.

While infected with COVID-19, their vertebral pain increased dramatically (from VAS $0.25 \pm 0.5$ to VAS $6.25 \pm 0.957, d=0.887)$. Pain intensity mostly resolved after COVID-19 recovery (VAS $0.5 \pm 1.0$ ) (Table 1 ).

Table 1. VAS score in four patients prior to, during, and after COVID-19 infection.

\begin{tabular}{cccccc}
\hline Patient & Gender & Age (years) & Pre-COVID-19 VAS & Peri-COVID-19 VAS & Post-COVID-19 VAS \\
\hline 1 & M & 32 & 1 & 5 & 0 \\
2 & F & 38 & 0 & 7 & 0 \\
3 & M & 43 & 0 & 6 & 2 \\
4 & M & 45 & 0 & 6 & 0 \\
\hline
\end{tabular}

F-female, M-male, VAS—visual analogue scale.

In the period of convalescence, vertebral algic syndrome and pseudoradicular irritation disappeared in three of the four patients, and they fully recovered. In one case, back pain of low intensity persisted and worsened after increased physical activity. Slow and continuous reduction of the lumbar back pain had been described by the patient himself during repeated examinations at the outpatient clinic.

\section{Discussion}

As shown, the vertebral pain was reported as the first complaint of COVID-19 in four physically active adults. Three patients had no previous history of vertebral algic syndrome or back pain. One patient complained of minor neck pain with no need for treatment. At the 
time of neurosurgical examination and during acute COVID-19 infection, the vertebral pain dramatically increased, whereas no other clinical manifestation of SARS-CoV-2 infection was present. The infection was coincidentally confirmed afterwards.

The course of COVID-19 was not severe; only symptomatic therapy was needed. Great variability and changes in intensity of the pain and cervical/lumbar pseudoradicular symptoms were present. The pain characteristics did not correspond with the findings of neuroimaging. Similarly to our clinical experiences, significant increases in musculoskeletal pain in COVID-19 patients during the course of disease in cervical (up to $46.6 \%$ ) and lumbar spine (to 50.7\%) were reported by Şahin et al. [27].

Extra-pulmonary musculoskeletal symptoms were commonly present among COVID-19 patients $[7,8,12,13,27]$. The central nervous system involvement has been studied on a deeper level, and some interesting hypotheses have been developed in published studies (headache, encephalitis, worsening of cognitive functions, onset/worsening of psychiatric disorders, Guillen-Barré syndrome, loss of taste and/or smell $[11,12,14])$, but the back pains remain unclear. The relationship between musculoskeletal pain and COVID-19 severity varied among the studies. While Chen and Zheng [6] reported that the occurrence of muscle pain was not associated with increased severity, Guan et al. [18] suggested that myalgias were an important predictive factor for the severity of the overall disease in patients with abnormal computed tomographic or radiographic imaging of the lungs.

When the disease resolved, the pain intensity dropped in a majority cases to the pre-infection level. However, other authors found that pain associated with COVID-19 could continue in the post-infection period [27] and could become chronic [11,13].

There is no question that an improved understanding of the rare symptoms of acute COVID-19 is needed. The viral load in patients with vertebral algic syndrome as the first/only symptom of SARS-CoV-2 infection could be similar to that of patients with asymptomatic or other manifestations of the novel coronavirus disease [28,29]. Therefore, closer attention should be paid by examining physicians.

Because clinical manifestations of novel coronavirus infection can be widely variable in clinical presentation and pain intensity, it seems impossible to distinguish SARS-CoV-2associated vertebral pain on the basis of clinical and radiological examination. The PCR test should be performed on suspicion of COVID-19-associated vertebral pain, especially in young, middle-aged, and physically active persons presenting acute onset of vertebral pain without a history of trauma. The mitigation of musculoskeletal pain in patients with COVID-19 might lead to further infection and unnecessary treatment.

The findings of this study have to be viewed in light of the limitation of its small sample size. The neurosurgical patients in our study most likely represent only the tip of the COVID-19-associated vertebral pain iceberg. This may be due to the fact that neurosurgeons are the last specialists to call for back pain. These results could be considered important for praxis in preventing the spread of SARS-CoV-2 among health care professionals and other patients, especially when other diagnostic procedures for back pain are required. Further investigation of this topic is needed.

\section{Conclusions}

Vertebral algic syndrome could be considered as the only sign of oligosymptomatic COVID-19 infection without respiratory tract manifestation and/or could preclude other clinical manifestations, especially in younger and middle-aged patients without previous back pain history. There is a pressing need for closer attention to be paid during the examination of patients presenting with the onset of new vertebral pain in the coronavirus pandemic.

If SARSCoV-2 infection is suspected, misdiagnosis or the delay of diagnosis should be avoided and the opportunity utilized to provide adequate treatment and prevent further transmission. The epidemiological history should not to be omitted, and the pros and cons of using personal protective equipment should be strictly considered, as this pain could be the first/only COVID-19 symptom of the patient. PCR confirmation of SARS- 
CoV-2 infection seems to be a necessary tool in the standard diagnostic process during the coronavirus pandemic. This case report adds another mosaic stone to our knowledge of COVID-19 as well as the manifestations of this disease.

Author Contributions: Conceptualization, J.C., A.D. and E.Z.; methodology, J.C. and A.C.; validation, J.C., A.D., R.I., A.C. and E.Z.; formal analysis, J.C. and A.C.; investigation, J.C.; resources, J.C., A.D. and R.I.; data curation, J.C.; writing—original draft preparation, J.C. and A.C.; writing-review and editing, J.C., A.C. and E.Z.; visualization, J.C.; supervision, E.Z. and A.D.; project administration, J.C.; funding acquisition, E.Z. All authors have read and agreed to the published version of the manuscript.

Funding: This research received no external funding.

Institutional Review Board Statement: The study was conducted in accordance with the principles outlined in the Helsinki Declaration. Ethical review and approval were waived for this study, for retrospective design of the routinely obtained data analysis and informed consent obtained.

Informed Consent Statement: Informed consent was obtained from all subjects involved in the study.

Data Availability Statement: The data presented in this study are available in the article.

Acknowledgments: This work was supported by the Cross-border Co-operation Programme INTERREG V-A SK-CZ/2018/06 (No. 304011P714) co-financed by the European Regional Development Fund.

Conflicts of Interest: The authors declare no conflict of interest.

\section{References}

1. Timeline: WHO's COVID-19 Response. Available online: https://www.who.int/emergencies/diseases/novel-coronavirus-2019 /interactive-timeline\#! (accessed on 24 June 2021).

2. John Hopkins Coronavirus Research Center. Available online: https:// coronavirus.jhu.edu/map.html (accessed on 23 June 2021).

3. Huang, C.; Wang, Y.; Li, X. Clinical Features of Patients Infected with 2019 Novel Coronavirus in Wuhan, China. Lancet 2020, 395, 497-506. [CrossRef]

4. Chen, N.; Zhou, M.; Dong, X. Epidemiological and Clinical Characteristics of 99 Cases of 2019 Novel Coronavirus Pneumonia in Wuhan, China: A descriptive study. Lancet 2020, 395, 507-513. [CrossRef]

5. Wang, D.; Hu, B.; Hu, C. Clinical Characteristics of 138 Hospitalized Patients With 2019 Novel Coronavirus-Infected Pneumonia in Wuhan, China. JAMA 2020, 323, 1061-1069. [CrossRef] [PubMed]

6. Chen, X.; Zheng, F. Epidemiological and Clinical Features of 291 Cases with Coronavirus Disease 2019 in Areas Adjacent to Hubei, China: A Double-Center Observational Study. Available online: https://www.medrxiv.org/content/10.1101/2020.03.03.2 0030353v1 (accessed on 3 May 2021).

7. Report of the WHO-China Joint Mission on Coronavirus Disease 2019 (COVID-19). Available online: https:/ /www.who.int/ docs/default-source/coronaviruse/who-china-joint-mission-on-covid-19-final-report.pdf (accessed on 25 February 2021).

8. Vetter, P.; Vu Diem, L. Clinical features of COVID-19. BMJ 2020, 369, m1470. [CrossRef]

9. Raveendran, A.V.; Jayadevan, R.; Sashidharan, S. Long COVID: An overview. Diabetes Metab. Syndr. 2021, 15, 869-875. [CrossRef]

10. Weng, L.; Su, X.; Wang, X. Pain Symptoms in Patients with Coronavirus Disease (COVID-19): A Literature Review. J. Pain Res. 2021, 14, 147-159. [CrossRef] [PubMed]

11. $\mathrm{Wu}, \mathrm{Y}$; $\mathrm{Xu}, \mathrm{X}$.; Chen, Z. Nervous system involvement after infection with COVID-19 and other coronaviruses. Brain Behav. Immun. 2020, 87, 18-22. [CrossRef]

12. Murat, S.; Dogruoz Karatekin, B. Clinical presentations of pain in patients with COVID-19 infection. Ir. J. Med. Sci. 2021, 190, 913-917. [CrossRef]

13. Cipollaro, L.; Giordano, L. Musculoskeletal symptoms in SARS-CoV-2 (COVID-19) patients. J. Orthop. Surg. Res. 2020, 15, 178-185. [CrossRef] [PubMed]

14. Giacomelli, A.; Pezzati, L. Self-reported Olfactory and Taste Disorders in Patients with Severe Acute Respiratory Coronavirus 2 Infection: A Cross-sectional Study. Clin. Infect. Dis. 2020, 7, 889-890. [CrossRef]

15. Aksan, F.; Nelson, E.A.; Swedish, K.A. A COVID-19 patient with intense burning pain. J. Neurovirol. 2020, 26, 800-801. [CrossRef] [PubMed]

16. Davis, K. COVID-19 pneumonia with back pain: Presentation of an acute pulmonary embolism associated with novel coronavirus infection in an outpatient setting. Clin. Case Rep. 2020, 8, 2514-2517. [CrossRef] [PubMed]

17. Marzano, A.V.; Cassano, N.; Genovese, G. Cutaneous manifestations in patients with COVID-19: A preliminary review of an emerging issue. Br. J. Dermatol. 2020, 183, 431-442. [CrossRef]

18. Guan, W.J.; Ni, Z.Y. China Medical Treatment Expert Group for COVID-19. Clinical characteristics of coronavirus disease 2019 in China. N. Engl. J. Med. 2020, 382, 1708-1720. [CrossRef] [PubMed] 
19. Sarkesh, A.; Daei Sorkhabi, A. Extrapulmonary Clinical Manifestations in COVID-19 Patients. Am. J. Trop Med. Hyg. 2020, 103, 1783-1796. [CrossRef] [PubMed]

20. Kucuk, A.; Cumhur Cure, M.; Cure, E. Can COVID-19 cause myalgia with a completely different mechanism? A hypothesis. Clin. Rheumatol. 2020, 39, 2103-2104. [CrossRef]

21. Desforges, M.; Le Coupanec, A.; Dubeau, P. Human Coronaviruses and Other Respiratory Viruses: Underestimated Opportunistic Pathogens of the Central Nervous System? Viruses 2019, 12, 14. [CrossRef]

22. Das, G.; Mukherjee, N.; Ghosh, S. Neurological Insights of COVID-19 Pandemic. ACS Chem. Neurosci 2020, 11, 1206-1209. [CrossRef]

23. Crichton, N. Visual analogue scale (VAS). J. Clin. Nurs. 2001, 10, 706-6.

24. Cohen, J. Statistical Power Analysis for the Behavioral Science; Lawrence Erlbaum Associates: Hillsdale, NJ, USA, 1988 ; pp. $19-66$.

25. Faul, F.; Erdfelder, E. G*Power 3: A flexible statistical power analysis program for the social, behavioral, and biomedical sciences. Behav. Res. Methods 2007, 39, 175-191. [CrossRef]

26. Booth, F.W.; Lees, S.J. Physically active subjects should be the control group. Med. Sci. Sports Exerc. 2006, 38, 405-406. [CrossRef]

27. Şahin, T.; Ayyildiz, A. Pain Symptoms in COVID-19. Am. J. Phys. Med. Rehabil. 2021, 100, 307-312. [CrossRef] [PubMed]

28. Hoehl, S.; Rabenau, H.; Berger, A. Evidence of SARS-CoV-2 infection in returning travelers from Wuhan, China. N. Engl. J. Med. 2020, 382, 1278-1280. [CrossRef] [PubMed]

29. Zou, L.; Ruan, F.; Huang, M. SARS-CoV-2 viral load in upper respiratory specimens of infected patients. N. Engl. J. Med. 2020, 382, 1177-1179. [CrossRef] [PubMed] 